\title{
Study on Bottom Topographical Measurement in Water Areas
}

\author{
Kazuhiro Yamamoto ${ }^{1}$ \\ Graduate School of Environmental and Life Science \\ Okayama University \\ Okayama City, Japan \\ e-mail: dev421102@s.okayama-u.ac.jp name@xyz.com \\ Narisu Chen ${ }^{3}$ \\ Graduate School of Environmental and Life Science \\ Okayama University \\ Okayama City, Japan \\ e-mail: chennarisu@gmail.com
}

\author{
Masaji Watanabe ${ }^{2}$ \\ Graduate School of Environmental and Life Science \\ Okayama University \\ Okayama City, Japan \\ e-mail: watanabe@ems.okayama-u.ac.jp
}

\author{
Hashentuya $^{4}$ \\ Graduate School of Environmental Science \\ Okayama University \\ Okayama City, Japan
}

\begin{abstract}
Techniques updating bottom topographical data are described. Positional data from a RTK-GPS are transformed to rectangular coordinates by the Gauss-Krüger Projection, and synchronized with depth data from an echo-sounder to generate three dimensional topographical data. A bottom topography over a triangular mesh is updated by two methods. Errors due to the movement of a boat are corrected by outcomes from a clinometers and a compass.
\end{abstract}

Keywords- RTK-GPS; Echo-sounder; Gauss-Krüger Projection; Clinometers; Compass; Finite element

\section{INTRODUCTION}

There is a finite element method in one of the numerical analysis method of flow in the hydrosphere. In the numerical analysis method, computational domain is required. However, with the passage of time, bottom topography in the hydrosphere, is changing by sedimentation and erosion. Therefore, it is necessary to reflect the computational domain to bottom topography latest. This study describes a case of applying to Kojima Lake updates of triangular elements used in the finite element method and the measurement of the bottom topography data. Update of triangular elements were applied two methods.

Features Kojima Lake is made as follows. Seto Inland Sea lies between the main island and the Shikoku Island of Japan, and Kojima Bay is situated in the coast of the Seto Inland Sea along the main island. A part of the Kojima Bay was isolated from the rest with embankment, and became a freshwater lake, Kojima Lake. Kojima Lake is approximately $10 \mathrm{~km} 2$ in area, and the primary source of the water is inflows from two rivers, Sasagase river and Kurashiki river. The water level of the Kojima Lake is controlled by drainage through six gates situated on the bank, which separates the Kojima Lake from the Kojima Bay, and the average water level is maintained at approximately $1.8 \mathrm{~m}$ to $2.1 \mathrm{~m}$ above the sea level. The gates are opened to drain water from the Kojima Lake to the Kojima Bay to maintain the water level of the lake when it is necessary. Lake is subject to change due to sedimentation and erosion. To investigate changes in the bottom topography of the Kojima Lake, positioning data from a RTK-GPS are transformed to rectangular coordinates by the Gauss-Krüger Projection, and synchronized with depth data from an echo-sounder to generate three dimensional bottom topographical data. Positioning data from a RTK-GPS are latitudinal, longitudinal, and altitudinal data recorded at every second. Longitudinal and latitudinal records are transformed with the Gauss-Krüger Projection to yield three dimensional trajectories. Those data are used to update a bottom topographical data over a triangular mesh [3 - 5].

Errors due to the movement of a boat such as roll, pitch, or yaw are corrected by outcomes from a clinometers and a compass. Experimental results are presented to validate our techniques. Figure 1 shows photos taken in the investigation conducted on October 25th, 2012. Figure 1 shows the rover antenna, the clinometers and the compass attached to a pole. The oscillator for the echo-sounder is attached to the end of the pole underneath the surface. Figure 2 shows the base station for the RTK-GPS.

\section{BOTTOM TOPOGRAPHY MEASUREMENT USING A RTK-GPS AND AN ECHO-SOUNDER}

Investigations were also conducted on April 14th, April 20th, May 15th, and November 26th, 2011 [4], and on June 22nd, and 29th, 2012 [5]. In this study, new outcomes from investigations conducted on October 11th and 25th, November 8th, and December 13th and 27th, 2012 are also introduced into analysis together with those obtained in previous studies. 


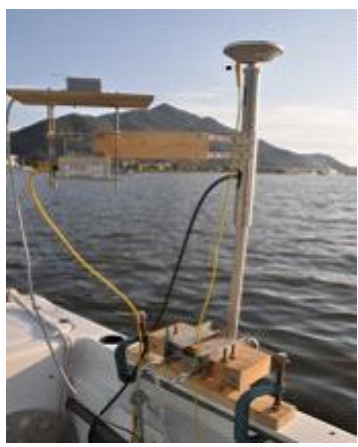

Figure 1. The rover antenna, the compass, and the clinometers. (Measurement conducted on October 25th, 2012.)[7].

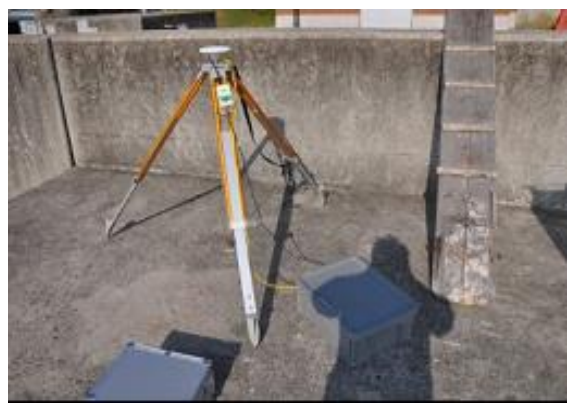

Figure 2. The base station [7].

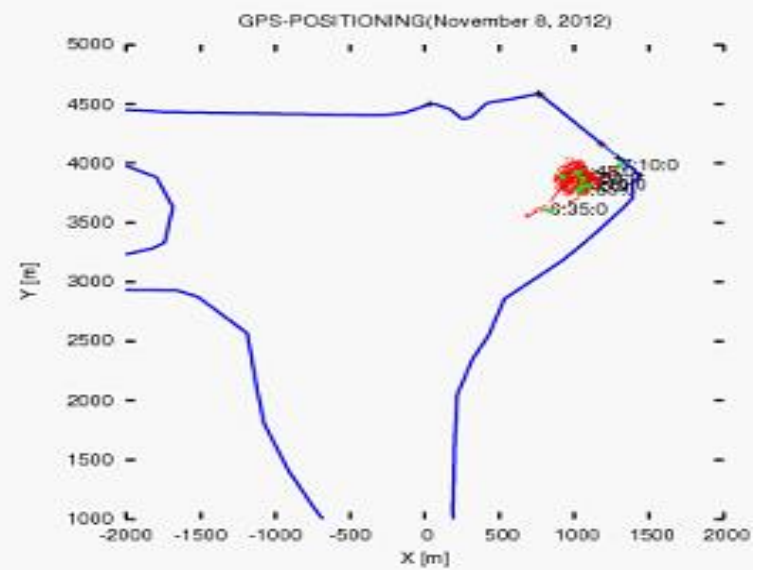

Figure 3. Wakes of the rover antenna. An outline of the Kojima Lake is also shown. (Outcomes from data recorded on November 8th, 2012[7].

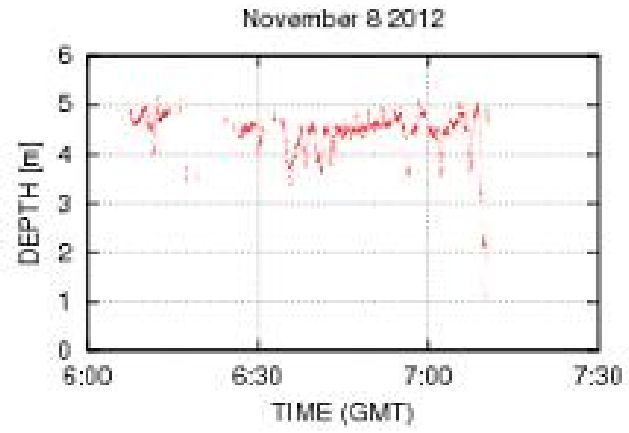

Figure 4. Time and echo-sounder data. (Outcomes from data recorded on November 8th, 2012.)[7].

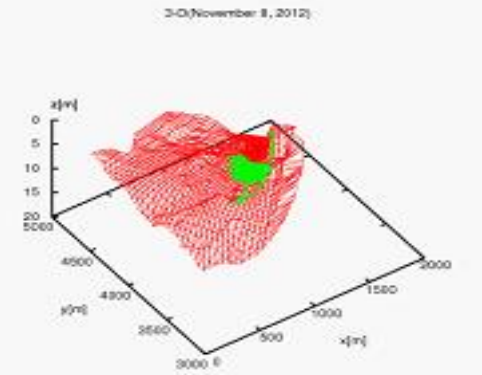

Figure 5. Three dimensional trajectory. A wire frame of the bottom topography before update is also shown. (Outcomes from data recorded on November 8th, 2012.)[7].

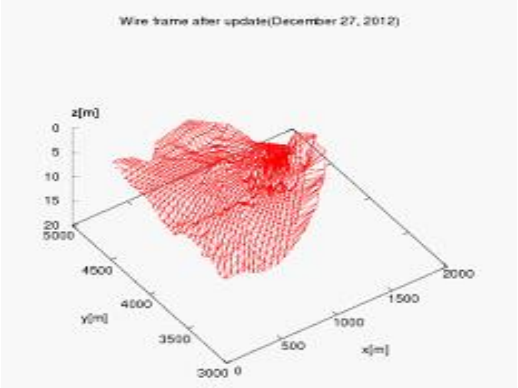

Figure 6. Wire frame after update. The figure shows the wire frame for the bottom topography the update using the data shown in Figure 2 - 6, the

October 11th and 25th, December 13th and 27th, 2012 data and the obtained in the previous studies $[4,5,7]$

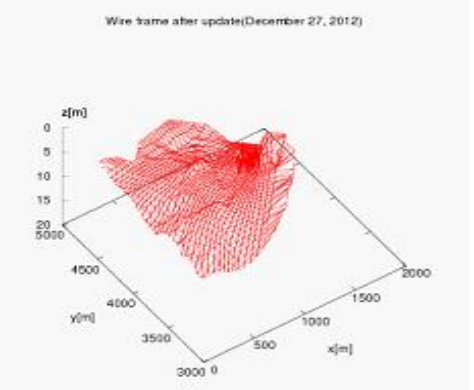

Figure 7. Wire frame after update by iterative calculation. The figure shows the wire frame for the bottom topography the update using the data shown in Figure 2 - 6, the October 11th and 25th, December 13th and 27th, 2012 data and the obtained in the previous studies [4, 5,7].

\section{UPDATE OF BOTTOM TOPOGRAPHY}

Techniques to update the topographical data over a finite element mesh using depth - position data were developed in previous studies $[4,5]$. The three dimensional data shown in Figure 5 were introduced into analysis to update the bottom topography of the Kojima Lake. The results are shown in Figure 6, 8 and 9. Figure 7 shows a wire frame of the bottom topography over a finite element mesh after update, Figure 8and 9 shows a comparison between the bottom topography data before and after the update. Furthermore, for the method of in previous study [4], add nodes of triangular elements in the three dimensional topographical data, and updating the triangular elements. The results are shown in Figure 7 and 10 
by iterative calculation. Figure 7 shows a wire frame of the bottom topography over a finite element mesh after update, and Figure 10 shows a bottom topography data after the update.

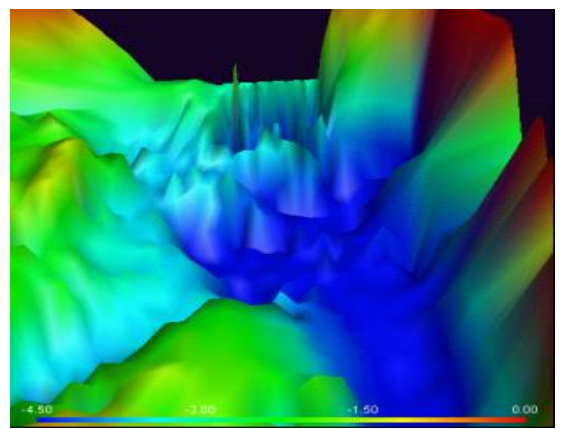

Figure 8. Before update [7].

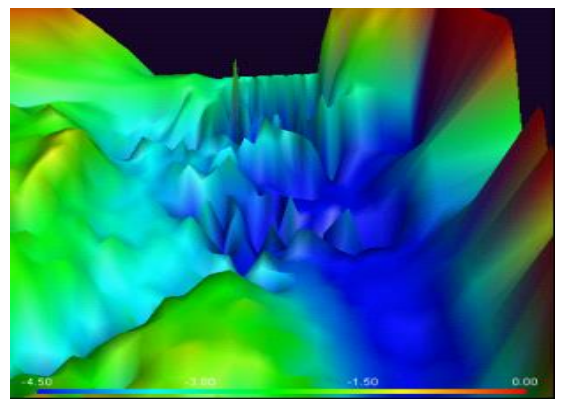

Figure 9. After update[7].

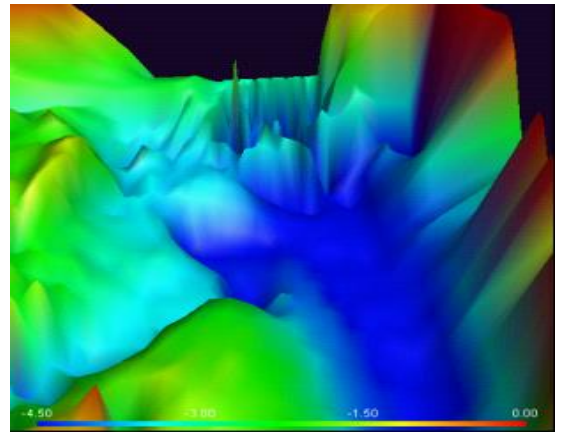

Figure 10. After update by iterative calculation[7].

\section{OBSERVE THE CHANGE OF BOTTOM TOPOGRAPHY IN THE NEAR THE GATE}

Figure 11 shows a new mesh that was created by finely divided the original mesh. The red mesh is original mesh, and blue mesh is new mesh. Measurement data were applied to update the bottom topography data on April 14th, April 20th, May 15th, and November 26th, 2011 [4], and on June 22nd, and 29th, and October 11th and 25th, November 8th, and December 13th and 27th, 2012. The results are shown in figures 12 and 13. Figure 13shows wire frame after update. The figure shows the wire frame for the bottom topography the update. The red dot is the measurement data. The bottom topography was became shallowed in the region near the gate. Because the gate is closed, and the flow is stagnant, precipitated.
A field measurement was conducted on November 13. 2013. Result of the measurement is show in Figure 14 with the bottom topography show in Figure 13 .

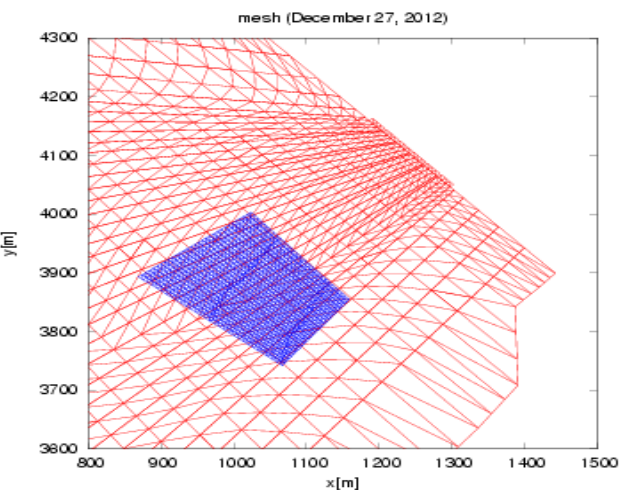

Figure 11. Blue mesh is finely divided the mesh.

mesh of after update (Dece mber 27, 2012)

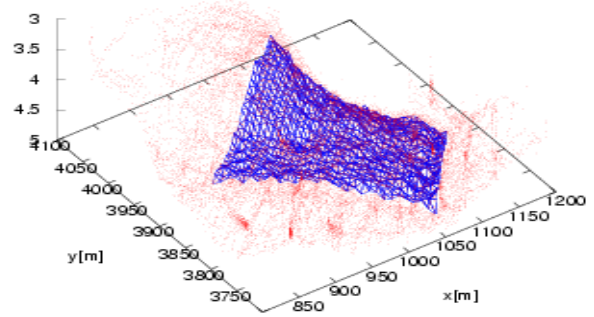

Figure 12. Wire frame after update. The figure shows the wire frame for the bottom topography the update.The red dot is the measurement data.(Outcomes from data recorded on December ,27h, 2012.)

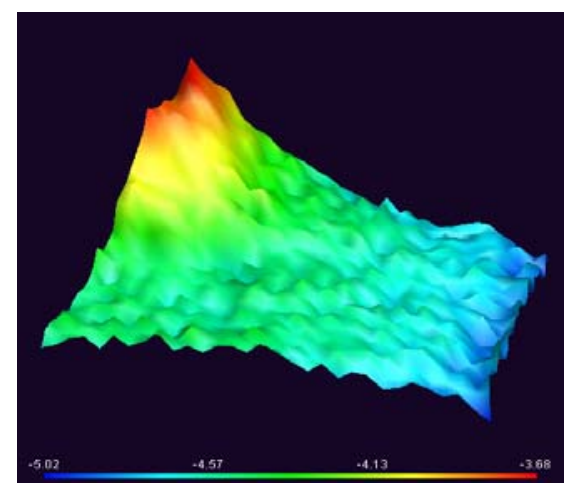

Figure 13. After update. 


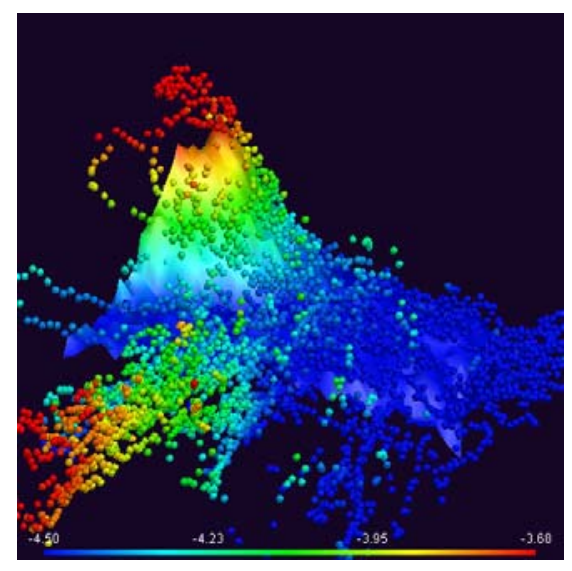

Figure 14. Results of Measurement in November 13,2013.

\section{CONCLUSION}

Accurate topographical data are essential for analyses of flows generated by the discharge of water from the lake $[1,2]$.In this study, this result described that techniques to update bottom topographical date and to correct errors due to the movement of a boat. Towards the update result of the triangular elements that introduced iterative, to obtain noticeable results. Experimental results to examine our techniques are also presented.The bottom topography was became shallowed in the region near the gate. Because the gate is closed, and the flow is stagnant, precipitated.

\section{ACKNOWLEDGMENT}

The authors are grateful to those people in the Bay Central Administration Office, the Section of Land Improvement in the Kojima Bay Area, those people in the Okayama Prefecture Okayama Development Bureau, those people in the Engineering Workshop, Center for Engineering [8]
Innovation, the Faculty of Engineering, Okayama University, and those people who assisted in the field measurement for their assistance. The information for generating the figures concerning the Kojima Lake is partially based on maps (C) Shobunsha Publications, INC., (C) Computer Graphic CO., LTD. This study was supported by Wesco Scientific Promotion Foundation.

\section{REFERENCES}

[1] Shinya Sumida, Ying Liu, Yu Li, Kazuhiro Yamamoto, Majda Ceric, Masaji Watanabe, Numerical study of currents generated in a lake, and verification by experiment, Proceedings of the 8th Biennial Engineering Mathematics and Applications Conference, EMAC-2007, Editors: Geoffry N. Mercer and A. J. Roberts, ANZIAM J., 49, C541 - C558, May 2008.

[2] Ying Liu, Kazuhiro Yamamoto, Majda Ceric, Masaji Watanabe, Numerical study of flow generated in a lake and experiment using GPS, ANZIAM J. 50:C912 - C929, June 2009.

[3] Kazuhiro Yamamoto, Hashentuya, Yoji Otani, Masaji Watanabe, Bottom Topography Measurement using RTK-GPS and High Precision Echo Sounder, CICC-ITOE 2011. Volume 2:309-312, 2011, 978-1-4244-9734-8/11/\$26.00 (c) 2011 IEEE

[4] Yamamoto, Hashentuya, Y. Otani, Y. He, M. Watanabe, Study on a bottom topography of a lake using positioning data and depth data, ANZIAMJ.,53(EMAC2011),C525C538.http://journal.austms.org.au/o js/index.php/ANZIAMJ/article/view/5110.

[5] K. Yamamoto, Hashentuya, Y. Otani, Y. He, M. Watanabe, Study on a bottom topography of a lake using positioning data and depth data, ANZIAMJ.53(EMAC2011),C525-C538. http://journal.austms.org.au/ojs/index.php/ANZIAMJ/article/view/51 10 Kazuhiro Yamamoto, Hashentuya, Yoji Otani, YiwenHe.

[6] Kazuhiro Yamamoto, Narisu Chen, Masaji Watanabe, Study on making of three-dimensional bottom topography data and precision inspection experiment of the measurement system, PROCEEDINGS,2012 International Conference of Information Science and Computer Applications (ICISCA 2012),November19th20th,2012, Bali Dynasty .

[7] Kazuhiro Yamamoto, Measurement of three dimensional bottom topographical data with consideration for movement of a boat and updating data on a finite element mesh, Doctoral dissertation, Okayama University, 2013. 